\title{
Symmetrical Josephson vortex interferometer as an advanced ballistic single-shot detector
}

\author{
I. I. Soloviev, ${ }^{1,2,3, a)}$ N. V. Klenov, ${ }^{4,2,3}$ S. V. Bakurskiy, ${ }^{4,5}$ A. L. Pankratov, ${ }^{6,7,3, b)}$ \\ and L. S. Kuzmin $8,3,1$ \\ ${ }^{1}$ Skobeltsyn Institute of Nuclear Physics, Lomonosov Moscow State University, 119991 Moscow, Russia \\ ${ }^{2}$ Lukin Scientific Research Institute of Physical Problems, 124460 Zelenograd, Moscow, Russia \\ ${ }^{3}$ Laboratory of Cryogenic Nanoelectronics, Nizhny Novgorod State Technical University n.a. R.E. Alekseev, \\ 603950 Nizhny Novgorod, Russia \\ ${ }^{4}$ Physics Department, Lomonosov Moscow State University, 119991 Moscow, Russia \\ ${ }^{5}$ Faculty of Science and Technology and MESA+, Institute for Nanotechnology, University of Twente, \\ 7500 AE Enschede, The Netherlands \\ ${ }^{6}$ Institute for Physics of Microstructures of RAS, 603950 Nizhny Novgorod, Russia \\ ${ }^{7}$ Lobachevsky State University of Nizhni Novgorod, 603950 Nizhny Novgorod, Russia \\ ${ }^{8}$ Chalmers University of Technology, SE-41296 Goteborg, Sweden
}

(Received 2 August 2014; accepted 29 October 2014; published online 19 November 2014)

\begin{abstract}
We consider a ballistic detector formed in an interferometer manner which operational principle relies on Josephson vortex scattering at a measurement potential. We propose an approach to symmetrize the detector scheme and explore arising advantages in the signal-to-noise ratio and in the back-action on a measured object by means of recently presented numerical and analytical methods for modeling of a soliton scattering dynamics in the presence of thermal fluctuations. The obtained characteristics for experimentally relevant parameters reveal practical applicability of the considered schemes including possibility of coupling with standard digital rapid single flux quantum circuits. (C) 2014 AIP Publishing LLC. [http://dx.doi.org/10.1063/1.4902327]
\end{abstract}

Ballistic detectors are widely used for mesoscopic quantum measurements. ${ }^{1}$ In these detectors, a measured system controls a transport of particles by creating a scattering potential. The detector scheme can be organized in an interferometer manner. For example, in the ballistic read-out of superconducting flux qubit, the scheme contains two equal Josephson transmission lines (JTLs), one of which is coupled to the qubit, ${ }^{2}$ see Fig. 1(a). Fluxons propagating simultaneously along the JTLs serve as particles in this scheme. The fluxon scattering at the current dipole induced by the qubit magnetic field (see Fig. 1(b)) provides measurable time delay between the moments of arrival of the fluxons to the end of the JTLs. This single-shot, non-projective measurements ${ }^{3,4}$ can be made nearly non-demolition by matching the measurement frequency with the frequency of coherent qubit oscillations as described in Ref. 2. Such read-out attracts interest in context of quantum computing which underwent rapid development in the last decade. ${ }^{5-9}$

A number of works were devoted to theoretical and experimental study of the detector. ${ }^{10-15}$ It appears that the main drawbacks in its operation come from relativistic effects of the fluxon dynamics. In the experiment, ${ }^{13,14}$ the authors used single annular JTL coupled to the qubit instead of a couple, measuring deviation of the fluxon rotation frequency. The measurement results show that this deviation does not depend on the measured magnetic field orientation (the current dipole polarity). The qualitative explanation is that the relativistic fluxon characteristic size becomes much smaller than the dipole length due to Lorenz contraction. Therefore, the total contribution of the successive scatterings at the dipole poles to

\footnotetext{
a)isol@phys.msu.ru
}

b)alp@ipmras.ru the frequency shift is independent on the order of the poles. Since the fluxon being inside the coupling loop induces circulating current affecting the qubit, the contraction additionally enhance the back-action. While slow fluxon is obviously preferred to gain the time response, ${ }^{11,12,15}$ the corresponding bias current, acting as the fluxon driving force, appears to be unreasonable for the experiment. ${ }^{12,13}$

In this work, we study two ways to overcome the discussed drawbacks and show that symmetrization of the coupling leads to significant increase of the detector signalto-noise ratio (SNR) making it independent on the fluxon velocity, and drastically decreases the back-action on the qubit. At the end of the paper, we discuss possibility of using the considered schemes with rapid single flux quantum (RSFQ) cells, allowing implementation of efficient interface between superconducting qubits and room temperature electronics on a basis of mature digital RSFQ technology.

Natural approach allowing the distinguishing of the current dipole polarity is a shrinking of the dipole length providing the dynamics of the first scattering affecting the second one. ${ }^{12}$ This simultaneously decreases the time response and the back-action. The current dipole is oriented collinearly to the direction of the fluxon propagation (see Figs. 1(a) and 1(b)), and so the fluxon velocity and the dipole length are the paired optimization parameters.

Here, we propose another approach to overcome the drawbacks. The idea is to make each fluxon scattering at one pole of the dipole. This can be realized by symmetrical connection of the coupling loop to the both detector JTLs as it is shown in Figs. 1(c) and 1(d). Since the both fluxons are involved in the scatterings, the detector response is effectively increased. The dipole polarity can be easily distinguished by the sign of the response. The back-action in this 
(a)

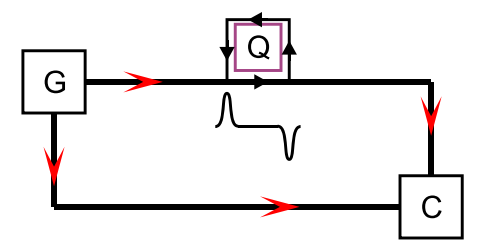

(b)

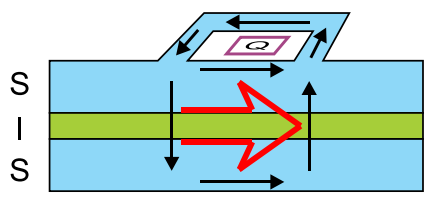

(c)

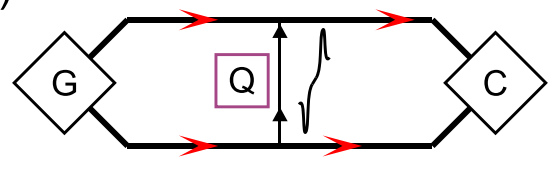

(d)

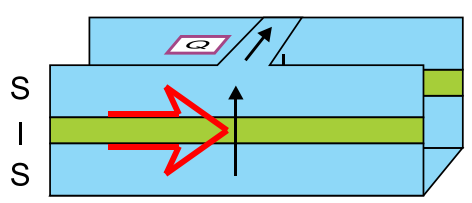

FIG. 1. (a) Ballistic Josephson vortex interferometer scheme. G box represents a fluxon generator, Q box-a qubit, and C box-a comparator. (b) Crosssectional scheme of continuous JTL coupled with a qubit. (c) The detector scheme in which the coupling loop is connected symmetrically to the both JTLs. (d) Cross-sectional scheme of the proposed symmetrical coupling. S denotes superconductor and I-isolator. The black arrows show current induced by the qubit magnetic field, the red arrows show the direction of the fluxon propagation.

scheme corresponds to the gained mutual shift of the fluxons and thus is proportional to the output signal. Thanks to the "orthogonal" orientation of the current dipole relatively to the direction of propagation of the fluxons, the only optimization parameter here is the fluxon velocity.

Estimation of the SNRs of the two considered detector schemes can be obtained using the modeling of the fluxon scattering dynamics in the frame of the sine-Gordon (SG) equation $^{16}$

$$
\phi_{t t}-\phi_{x x}+\sin (\phi)=-\alpha \phi_{t}+i+i_{f}(x, t)+i_{s}(x),
$$

where the space coordinate $x$ and the time $t$ are normalized to the Josephson penetration length $\lambda_{J}$ and to the inverse plasma frequency $\omega_{p}^{-1}$, respectively, $\alpha=\omega_{p} / \omega_{c}$ is the damping coefficient, $\omega_{p}=\sqrt{2 e I_{c} / \hbar C}, \omega_{c}=2 e I_{c} R_{N} / \hbar, I_{c}$ is the critical current, $C$ is the JTL capacitance, and $R_{N}$ is the normal state resistance. The current densities $i, i_{f}, i_{s}$ are normalized to the critical current density $J_{c} ; i$ denotes the dc overlap bias current density; $i_{f}$ is the fluctuational current density, which correlation function is $\left\langle i_{f}(x, t) i_{f}\left(x^{\prime}, t^{\prime}\right)\right\rangle=2 \alpha \gamma \delta$ $\left(x-x^{\prime}\right) \delta\left(t-t^{\prime}\right)$, where $\gamma=I_{T} / J_{c} \lambda_{J}$ is the dimensionless noise intensity, ${ }^{17,18} I_{T}=2 e k T / \hbar$ is the thermal current, $e$ is the electron charge, $\hbar$ is the Planck constant, $k$ is the Boltzmann constant, and $T$ is the temperature. $i_{s}(x)$ describes the current dipole. In the case of the collinear dipole orientation, this term is $i_{s}(x)=\mu_{\|}\left[\delta\left(x+d / 2-x_{c}\right)-\delta\left(x-d / 2-x_{c}\right)\right]$, with $\mu_{\|}=I_{p} M / 2 L_{c l} J_{c} \lambda_{J}$, where $\pm I_{p}$ is the current circulating in the qubit when it is far from the symmetry point (the sign corresponds to the current direction), $M$ is the mutual inductance between the qubit and the coupling loop, $L_{c l}$ is the inductance of the coupling loop, $d$ is the dipole length, and $x_{c}$ is its central coordinate. In the case of orthogonal dipole orientation, the expression transforms to $i_{s}(x)=\mu_{\perp} \delta\left(x-x_{c}\right)$, where $\mu_{\perp} \simeq 2 \mu_{\|}{ }^{16}$ The dipole amplitude doubling comes from the fact that in the collinear case the current circulating in the coupling loop spreads into the two streams flowing through the top and the bottom JTL electrodes as it is illustrated in Fig. 1(b). The width of the coupling loop wire, defining the width of the injected current distribution, is assumed to be much smaller than $\lambda_{J}$ allowing the using of the Dirac delta function. Following the work, ${ }^{11}$ we also assumed that perturbation of the fluxon dynamics is governed by the term $i_{s}(x)$ expressed in the presented forms even if the qubit is prepared initially in the symmetry point. This is valid for relatively strong coupling, where the qubit can be adiabatically shifted far from the symmetry point by the moving fluxon.

In our works, ${ }^{19,20}$ it was shown that the jitter $\sigma$ (the standard deviation) of fluxon propagation time (with the mean $\tau$ ) through the JTL can be suppressed if the fluxon moves with an acceleration. Following Ref. 15, we will consider the case where initially resting fluxons accelerate to the stationary velocity: ${ }^{21} u_{s t}=1 / \sqrt{1+(4 \alpha / \pi i)^{2}}$ normalized to the Swihart velocity $c=w_{p} \lambda_{J}$. Besides numerical simulation of the fluxon dynamics using the Eq. (1), in the experimentally relevant case, where all parameters in the right-hand side of the Eq. (1) are small $\alpha, i, i_{f}, i_{s} \ll 1$, one can use the analytical approach developed for relativistic fluxon velocities (see Ref. 15). In the frame of this approach, the scatterings and the noise effect are considered as perturbations to the fluxon dynamics governed by the energy balance which is defined by the constant terms $i$ and $\alpha$ representing the energy gain and loss.

For the two considered dipole orientations, the detector time response $\Delta \tau=\left|\tau_{L}-\tau_{R}\right|$, its jitter $\sigma_{\Sigma}=\sqrt{\sigma_{L}^{2}+\sigma_{R}^{2}}$, and their ratio, which is assumed to be the SNR, versus the bias current $i$ are shown in Figs. 2(a)-2(c) (here " $L$ " and " $R$ " indexes denote left and right shoulders of considered interferometer). The data for the collinear and the orthogonal dipole orientations are marked by the signs $\|$ and $\perp$ correspondingly. In the collinear case, the dipole polarity (marked as positive "+" or negative "-") reflects orientation of the first pole relatively to the bias current. For the orthogonal case, the data are shown for only one dipole polarity for compactness, since the polarity inversion reverses only sign of the time response. The dipole oriented collinearly is located at the center of the JTL of the length $L=60$, spread over the distance $d=L / 3$. The orthogonal dipole is located in such a way that its central coordinate coincides with the coordinate of the first pole of the collinear dipole: $x_{c \perp}=x_{c \|}-d / 2$. The dipole amplitude is taken typical for the experiment: ${ }^{13,14} \mu_{\|}=\mu_{\perp} / 2=0.05$. The damping parameter $\alpha=0.01$ and the normalized noise intensity $\gamma=0.001$ are typical for tests at $4.2 \mathrm{~K}$ temperature. 
(a)

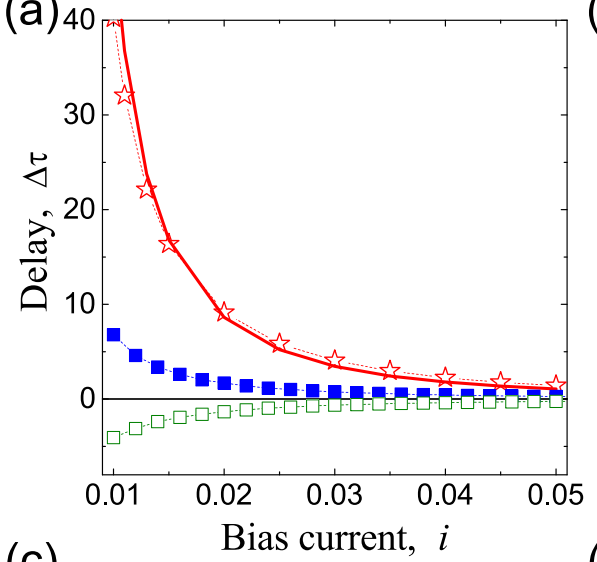

(c)

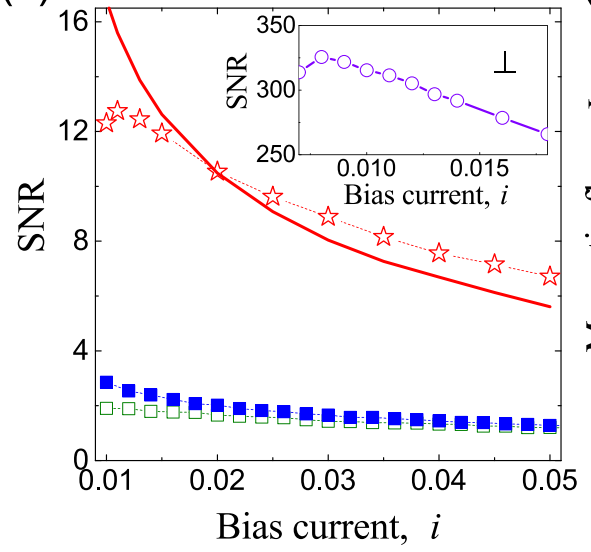

(b)

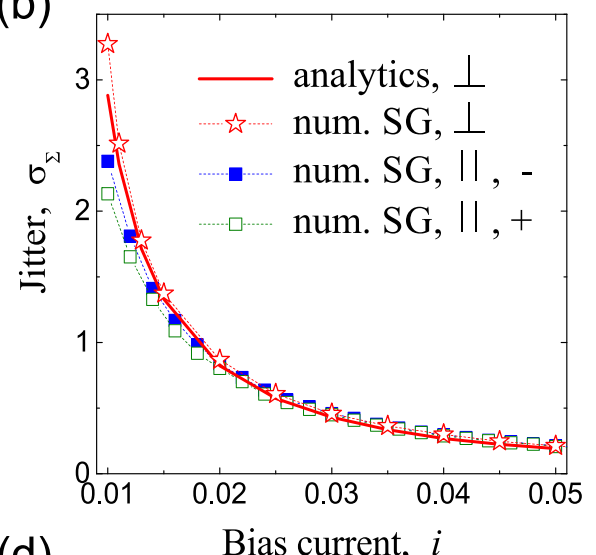

(d)

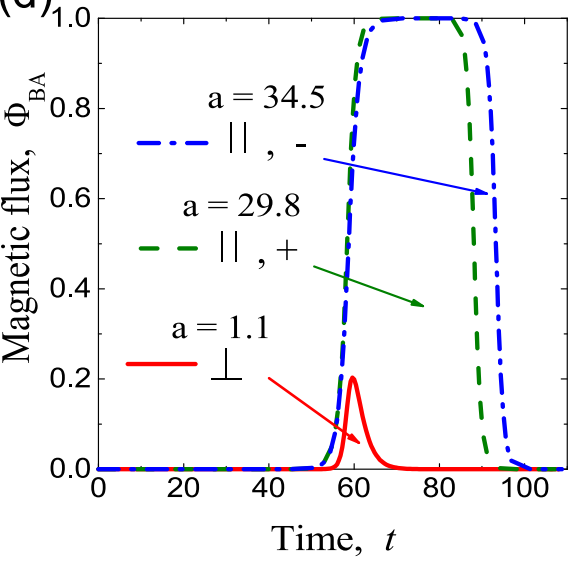

FIG. 2. (a) The detector time response, (b) its jitter, and (c) the SNR versus the bias current. $\alpha=0.01, \gamma=0.001$, $\mu_{\|}=\mu_{\perp} / 2=0.05, L=60, x_{c \|}=30$, $d=20, x_{c \perp}=20$. Inset of (c) presents the SNR dependence for $\alpha=0.001$ and $\gamma=10^{-5}$ for orthogonal orientation of the dipole. (d) The back-action flux versus time for the same parameters of the schemes and $i=0.02$.
The bias current decrease makes the dipole effect more pronounced decreasing velocities of the fluxons, see Fig. 2(a). Figs. 2(b) and 2(c) illustrate that the latter finally leads to significant jitter increase ${ }^{19,20}$ which becomes especially enhanced in the vicinity of the threshold bias current, leading to the fluxon capturing by the dipole in some realizations, that manifests itself as the SNR curve bend. Though this effect can not be traced using the analytical approach (the corresponding data are shown by the solid curves), the SNR estimation for larger bias currents is consistent with the results of numerical simulations (obtained with averaging over 10000 realizations, presented by dotted curves) revealing the SNR enhancement due to the symmetrization by the factor of 5 . For the optimal bias current range, the symmetrical scheme possesses the SNR well above 100, see the inset of Fig. 2(c) for parameters corresponding to $T<100 \mathrm{mK}$ temperature range. Since the damping in real experiment depends on the specific tunnel resistance of a long Josephson junction, one would expect a decrease of one order of magnitude ${ }^{10}$ in the dissipation parameter compared to $T=4.2 \mathrm{~K}$, so $\alpha=0.001$. At the same time, if the temperature is not too low $T \geq 50 \mathrm{mK}$, so that the thermal noise is predominant, ${ }^{22}$ then the normalized noise intensity scales proportional to the temperature down to $\gamma \simeq 10^{-5}$.

The flux $\Phi_{c f}$ captured by the coupling loop in the two considered cases can be calculated as convolution of the according function $F_{c l \|}$ or $F_{c l \perp}$ representing the coupling loop placement in the detector (corresponding to the $i_{s}(x)$ ) with the magnetic flux of the vortex. The flux affecting the qubit $\Phi_{B A}$ is then expressed as follows:

$$
\begin{gathered}
\Phi_{B A \|}=\frac{M}{L_{c l}} \Phi_{c f}=\frac{M}{L_{c l}}\left(F_{c l \|} * \Phi_{f l}\right)(X), \\
\Phi_{B A \perp}=\frac{M}{L_{c l}}\left[\left(F_{c l \perp} * \Phi_{f l 1}\right)\left(X_{1}\right)-\left(F_{c l \perp} * \Phi_{f l 2}\right)\left(X_{2}\right)\right], \\
\Phi_{f l}=\frac{4 e^{\theta}}{\left(1+e^{2 \theta}\right) \sqrt{1-u^{2}}},
\end{gathered}
$$

where $F_{c l \|}(x)=H\left(x+d / 2-x_{c}\right)-H\left(x-d / 2-x_{c}\right)$ and $F_{c l \perp}(x)=H\left(x-x_{c}\right), H$ is the Heaviside step function, $X$ and $u$ are the central coordinate of the fluxon and the fluxon's velocity which can be found using the approaches presented in Ref. $15, \theta=(x-X) / \sqrt{1-u^{2}}$, and $\Phi_{f l}$ is the vortex magnetic flux distribution.

The back-action flux dependences on the time are shown in Fig. 2(d). Assuming that probability for non-adiabatic transitions is small, ${ }^{12}$ we can consider an integral influence of the flux as a quantitative characteristic of back-action. The qubit phase perturbation can be expressed as follows: $\delta \varphi=\int_{0}^{\tau} \delta E_{0}(t) d t / \hbar$ with $\delta E_{0}(t)$ being the deviation of the qubit energy level splitting dependent on time due to influence of the back-action flux. ${ }^{12}$ Therefore, the definite time integral $a=\int_{0}^{\tau} \Phi_{B A}(t) d t$ is a comparison parameter for qualitative estimation of the back-action. It is seen that the symmetrization provides significant back-action reduction because of differential origin of the back-action flux.

Fig. 2(c) shows that the SNR can be rather small in the case of collinear dipole orientation in the detector. It can be increased by increase of the qubit coupling strength (the dipole amplitude $\mu$ ) or by placement of the dipole poles close to the ends of the JTL. The latter provides the first scattering 


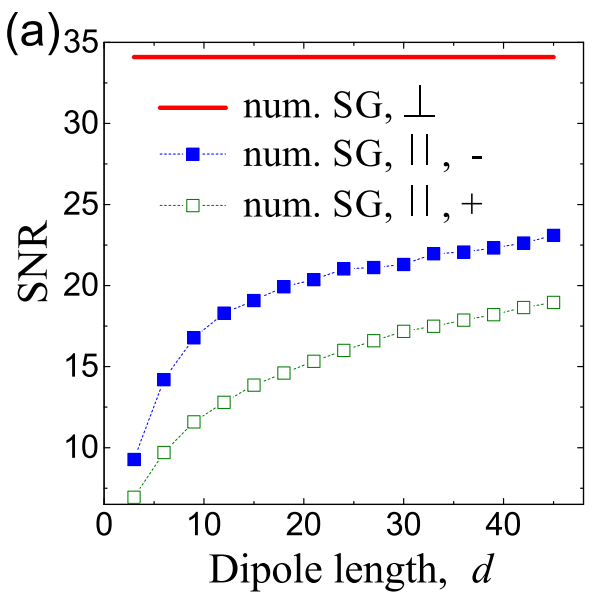

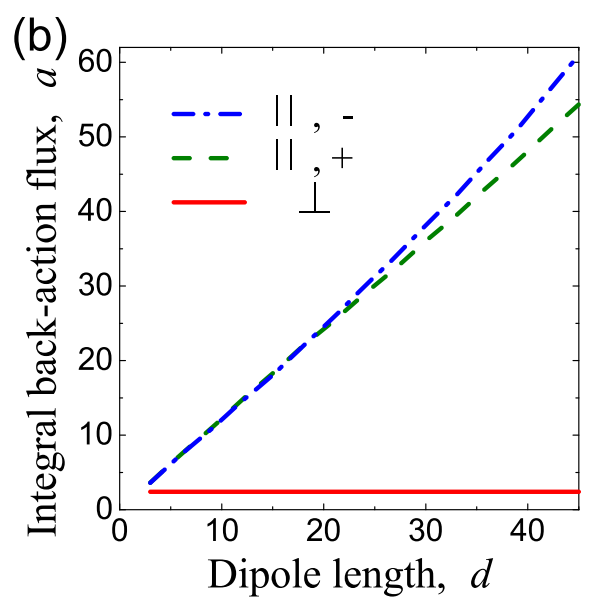

FIG. 3. (a) The SNR and (b) the integral back-action flux versus the current dipole length, $\alpha=0.01, \quad i=0.046$, $\gamma=0.001, \quad \mu_{\|}=\mu_{\perp} / 2=0.2, \quad L=60$, $x_{c 1 \|}=5, x_{c \perp}=8$. occurring at low fluxon velocity and suppression of the damping effect of fluxon scattering at the second dipole pole. However, the both approaches increase the back-action. We found the optimal collinear dipole placement at $d \simeq 15-20$ by fixing it first pole near the beginning of the JTL: $x_{c 1 \|}=5$ and varying the dipole length at the optimal bias current value $i=0.046$, see Fig. 3 . For the shorter dipole length, the poles start to compensate each other, while for the larger ones the integral back-action flux begins to grow nonlinearly. For the orthogonal dipole case, the data are shown by solid lines. The symmetrical scheme clearly possesses the superior characteristics.

In Ref. 14, it was argued that with the progress in superconducting quantum computing, a lot of data will be processed at low temperatures, and therefore the using of mature digital RSFQ electronics ${ }^{23}$ as an interface between quantum circuits and room temperature electronics is quite natural. The considered schemes can be easily incorporated with RSFQ circuits, since they imply single-shot measurements and can be implemented on nearly the same element basis as RSFQ cells, i.e., using discrete JTLs. In this case, one can use standard RSFQ circuits improving overall interface characteristics, e.g., increasing the time resolution of the output detector comparator using RSFQ time vernier ${ }^{11}$ or simultaneously reading-out several coupled qubits ${ }^{16}$ using RSFQ splitter tree. ${ }^{24}$

Fig. 4 presents the SNR of the detector with orthogonal dipole orientation versus the bias current, the dipole amplitude, its central coordinate, and the noise intensity. The value of the normalized inductance coupling lumped Josephson junctions in the discrete detector JTLs is $l=2 e L_{c j} I_{c j} / \hbar=1$, where $L_{c j}$ is the absolute inductance value and $I_{c j}$ is the critical current of the junction. For the low damping case, the bias current increase leads to the transition of the system from the "locked" to the "running phase" mode, considered in Ref. 20, which is accompanied by multiple creations of fluxon-antifluxon pairs and manifests itself by abrupt SNR growth that is seen in Fig. 4(a) for $i \simeq 0.5$. For the low bias current values, e.g., around $i=0.1$, the system is in the conventional "locked" mode (corresponding to single fluxon propagation) with working bias current margins well exceeding the standard RSFQ margins $\pm 25 \%$. Inset of Fig. 4(b) shows that for the chosen bias current value $(i=0.1)$ and found optimal dipole placement $x_{c \perp}=7$ (see the SNR versus $x_{c \perp}$ curves in Fig. 4(b)) at the noise intensity value $\gamma=10^{-5}$, the detector SNR can be as high as $S N R=600$ for $\alpha=0.01$ and $S N R=1800$ for $\alpha=0.001$. If these values are excessive, they allow the decreasing of the back-action by reducing the strength of the qubit coupling (see the SNR versus $\mu$ dependence in the inset of Fig. 4(a)) or the increasing of the working bias current margins by increasing the damping (the SNR curves for different damping values are shown in Fig. 4(a)).

In conclusion, we optimized the Josephson vortex interferometer in respect to the SNR and the back-action tradeoff. We have shown that symmetrization of the scheme leads to significant SNR increase due to the involvement of the both fluxons in the scattering events and effective increase of the scattering potential amplitude. At the same time, the
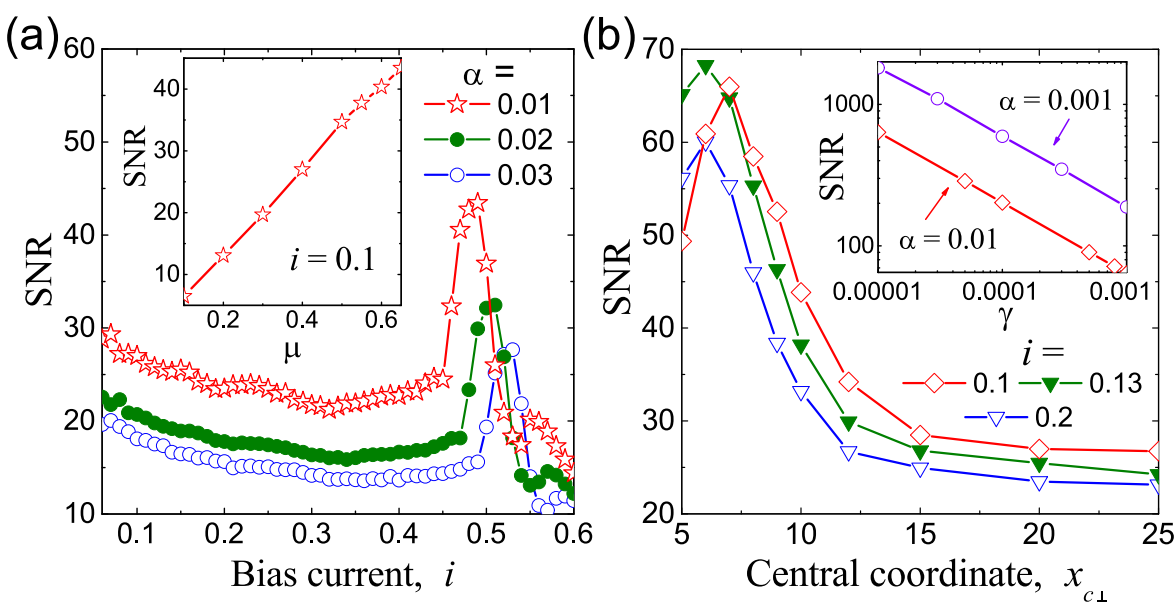

FIG. 4. (a) The SNR versus the bias current at different values of the damping. $\gamma=0.001, \mu_{\perp}=0.4, x_{c \perp}=20$. Inset shows the SNR versus the current dipole amplitude for $\alpha=0.01$ and $i=0.1$. (b) The SNR versus the dipole position at different bias current values, $\alpha=0.01$, $\gamma=0.001, \mu_{\perp}=0.4$. Inset shows the SNR versus the noise intensity for $i=0.1, x_{c \perp}=7, \alpha=0.01,0.001$. The length of the discrete JTLs is: $L=40$ and $l=1$. 
back-action is drastically reduced because of differential origin of the back-action flux. The SNR estimation for the both cases of utilization the continuous and the discrete JTLs, for experimentally relevant parameters, is well above 100. This opens the opportunity of using the considered detector in practical applications including implementation of a unified interface circuit on the basis of RSFQ digital cells for linking the room temperature electronics and it quantum superconducting counterparts.

The authors would like to acknowledge fruitful discussions with E. Il'ichev. This work was supported by Russian Ministry of Science (Project Nos. 3.2054.2014/K, 11.G34.31.0029, 14.604.21.0005, and 02.B.49.21.0003), RFBR Project No. 14-02-31002-mol_a, Russian President Grant No. MK-1841.2014.2, and Dynasty Foundation.

${ }^{1}$ D. Averin, e-print arXiv:cond-mat/0603802v1.

${ }^{2}$ D. V. Averin, K. Rabenstein, and V. K. Semenov, Phys. Rev. B 73, 094504 (2006).

${ }^{3}$ B. M. Mensky, Quantum Measurements and Decogerence: Models and Phenomenology (Kluwer Academic Publishers, 2000).

${ }^{4}$ V. B. Braginsky, Yu. I. Vorontsov, and K. S. Thorne, Science 209, 547 (1980).

${ }^{5}$ Y. Makhlin, G. Schön, and A. Schnirman, Rev. Mod. Phys. 73, 357 (2001).

${ }^{6}$ J. Q. You and F. Nori, Phys. Today 58(11), 42 (2005).
${ }^{7}$ J. Clarke and F. K. Wilhelm, Nature 453, 1031 (2008).

${ }^{8}$ A. A. Houck, H. E. Tureci, and J. Koch, Nat. Phys. 8, 292 (2012).

${ }^{9}$ M. H. Devoret and R. J. Schoelkopf, Science 339, 1169 (2013).

${ }^{10}$ A. Herr, A. Fedorov, A. Shnirman, E. Ilichev, and G. Schön, Supercond. Sci. Technol. 20, S450 (2007).

${ }^{11}$ A. Fedorov, A. Shnirman, G. Schön, and A. Kidiyarova-Shevchenko, Phys. Rev. B 75, 224504 (2007).

${ }^{12}$ K. G. Fedorov, Ph.D. thesis, Karlsruher Institute of Technology, 2013.

${ }^{13}$ K. G. Fedorov, A. V. Shcherbakova, R. Schäfer, and A. V. Ustinov, Appl. Phys. Lett. 102, 132602 (2013).

${ }^{14}$ K. G. Fedorov, A. V. Shcherbakova, M. J. Wolf, D. Beckmann, and A. V. Ustinov, Phys. Rev. Lett. 112, 160502 (2014).

${ }^{15}$ I. I. Soloviev, N. V. Klenov, A. L. Pankratov, E. Il'ichev, and L. S. Kuzmin, e-print arXiv:1409.2658.

${ }^{16}$ See supplementary material at http://dx.doi.org/10.1063/1.4902327 for consideration of perturbation terms in the SG equation and possibility of reading-out several coupled qubits using the proposed detector scheme.

${ }^{17}$ K. G. Fedorov and A. L. Pankratov, Phys. Rev. B 76, 024504 (2007).

${ }^{18}$ K. G. Fedorov and A. L. Pankratov, Phys. Rev. Lett. 103, 260601 (2009).

${ }^{19}$ A. L. Pankratov, A. V. Gordeeva, and L. S. Kuzmin, Phys. Rev. Lett. 109, 087003 (2012).

${ }^{20}$ I. I. Soloviev, N. V. Klenov, A. L. Pankratov, E. Il'ichev, and L. S. Kuzmin, Phys. Rev. E 87, 060901(R) (2013).

${ }^{21}$ D. W. McLaughlin and A. C. Scott, Phys. Rev. A 18, 1652 (1978).

${ }^{22}$ G. Oelsner, L. S. Revin, E. Ilichev, A. L. Pankratov, H.-G. Meyer, L. Grönberg, J. Hassel, and L. S. Kuzmin, Appl. Phys. Lett. 103, 142605 (2013).

${ }^{23}$ S. Nishijima, S. Eckroad, and A. Marian, Supercond. Sci. Technol. 26, 113001 (2013).

${ }^{24}$ V. K. Kornev, I. I. Soloviev, N. V. Klenov, and O. Mukhanov, Supercond. Sci. Technol. 19, S390 (2006). 